Revista Venezolana de Gerencia (RVG)

Año 11. № 33, 2006, 105 - 121

Universidad del Zulia (LUZ) • ISSN 1315-9984

\title{
El trabajo como fenómeno de autoorganización
}

\author{
Mendoza de Ferrer, Hermelinda* \\ Rodríguez P., María**
}

\section{Resumen}

La investigación tuvo como objetivo reflexionar acerca de los cambios ocurridos en el mundo del trabajo y si estos pueden ser considerados o no como fenómenos de autoorganización. El problema se abordó mediante una investigación documental con enfoque hermenéutico. Los resultados obtenidos muestran cómo la disipación es un fenómeno creativo que, en lugar de incrementar el desorden, hace surgir nuevas realidades no sometidas directamente al control de los sujetos que las producen lográndose formas elaboradas de estabilidad a partir del caos y la confusión, tal como ocurre en las nuevas formas de organización del trabajo. Se concluye que los desequilibrios ocurridos en el ámbito del trabajo devienen en nuevas formas de organización surgidas a partir del diálogo e intercambio en la relación sujeto-objeto capaz de producir la autoorganización para atender las complejas realidades del mundo de hoy.

Palabras clave: Trabajo, autoorganización, caos.

\section{Work as a Phenomenon of Self-Organization}

\begin{abstract}
The object of this research was to reflect on the changes that have occurred in the world of the labor, and whether or not they should be considered as phenomenon of self-organization. A documentary approach was adopted with an hermeneutic focus. The results points out that dissipation is a creative phenomenon, which instead of increasing the disorder, causes the upsurge of new realities, which are not submitted directly to the control of the subjects that produce them. In this manner elaborate forms of stability result from chaos and confusion, such as occurs in new forms of labor organizations. The conclusions are that the imbalances which occurred in the work environment become new forms of organization which arise from these organizations, based on dialogue and exchange in the relation subject-object, and are capable of producing self-organization in order to confront complex realities in the modern world.
\end{abstract}

Key words: Work, self-organization, chaos.

Recibido: 05-06-12. Aceptado: 06-02-13

* Profesora Asociada de la Escuela de Administración y Contaduría Pública de la Universidad de Carabobo. Acreditada en el Programa Promoción al Investigador (PPI).

** Profesora Asociada de la Escuela de Relaciones Industriales de la Universidad de Carabobo. Acreditada en el Programa Promoción al Investigador (PPI), nivel candidata. 
El trabajo como fenómeno de autoorganización

Mendoza de Ferrer, Hermelinda y Rodríguez P., María

\section{Introducción}

La sociedad vive una transición de la sociedad industrial a la sociedad postindustrial o del conocimiento (Drucker, 1993), de la modernidad a la postmodernidad (Schvarstein, 1998), de la simplicidad a la complejidad (Morin, 1990), (Franco y Dieterich, 1999), pudiéndose observar diferencias significativas en las ideas de uno y otro modelo civilizatorio, como también, la inminente necesidad de adecuarse a las nuevas exigencias, como condición indispensable para sobrevivir en un mundo caracterizado por la ambigüedad y la incertidumbre (Almarza, 2002), cuya única constante es un cambio general, acelerado y profundo.

Estos cambios son estimulados por la incorporación de la tecnología a los procesos productivos, la globalización de la economía y la dinámica de las estructuras sociales que buscan acoplarse a las nuevas exigencias. Los sistemas sociales en respuesta a los cambios, buscan de manera incesante el reacomodo producto del sentido emergente de las realidades, comportándose éstas como estructuras disipativas, que se mantienen en permanente movimiento y transformación, de allí que Prigogine citado por Martínez (1997: 128), para quien "la noción de realidad está indisolublemente ligada a los problemas del tiempo y del devenir", afirma lo siguiente "Cuanto más compleja sea una estructura disipativa, más energía necesita para mantener todas sus conexiones. Por ello también es más vulnerable a las fluctuaciones internas. Se dice entonces que esta más lejos del equilibrio. Debido a que estas conexiones solo pueden ser sostenidas por el flujo de energía, el sistema siempre esta fluyendo. Cuanto más coherente o intrincadamente conectada esté una estructura, más inestable es. Así al aumentar la coherencia se aumenta la inestabilidad. Pero esta inestabilidad es la clave de la transformación".

En atención a esta afirmación del autor, "el trabajo" se constituye en un hecho social complejo y estructuralmente disipativo, ya que en función de los procesos de transformación política, social, económica y cultural, entre otros, tiende a la autoorganización ${ }^{1}$ producto de la inestabilidad que les transmite el mismo hecho de ser realizado por personas huma-

$1 \quad$ La teoría de las estructuras disipativas de llya Prigogine plantea un modelo de transformación al explicar el papel de la tensión en el proceso de transformación y fuerza de movimiento existente en la naturaleza; a partir del principio de que nada está estático a nivel profundo sino en un movimiento continuo generador de fluctuaciones; si las fluctuaciones alcanzan un nivel preciso se incrementa el número de interacciones del sistema operándose nuevas conexiones, de esta manera, las partes se reorganizan para formar una nueva entidad de forma que el sistema adquiere un orden superior, más integrado y conectado que el precedente siendo, desde este punto de vista, la inestabilidad el punto focal de la transformación y las estructuras en desequilibrio, fenómenos de autoorganización (Martínez, 1997). 
nas que lo ubica como sistema dinámico complejo (Franco y Dieterich, 1999) ${ }^{2}$. En el caso de la disertación de este documento la noción de trabajo a la que se hace referencia, es la que tiene ver con el trabajo productivo, asalariado y que emerge producto de las revoluciones industriales asociadas con la manufactura y maquinismo.

En este orden de ideas, el hilo conductor de esta disertación esta enmarcado en la revisión de las formas de organización del trabajo a la luz de los sistemas productivos como noción histórica y los paradigmas que prevalecen y buscan explicar la realidad, para precisar las conexiones y fluctuaciones que se han venido dando en el devenir del hecho social trabajo, para esbozar las rasgos de autoorganización que se han dado en los modelos de gestión, los cuales buscan lograr el acoplamiento y transformación en las manera de hacer y producir, en función de los requerimientos de cambio que se plantean en el contexto.

\section{Consideraciones sobre el paradigma emergente}

El contexto de análisis se acopla con la dinámica del trabajo como fenómeno de autoorganización, en sintonía con los postulados del paradigma emergente, que permite, de forma incluyente, el abordaje de la realidad. En este orden de ideas Martínez (1997) considera que la ciencia occidental avanza hacia un paradigma llamado a cambiar la concepción de realidad actualmente vigente, como también, muchos aspectos de la naturaleza humana; por lo tanto, al confrontar la red de relaciones que ocurren dentro de una realidad particular y entre diversas realidades, se evidencia un conjunto de postulados que se constituyen en los elementos clave del paradigma emergente, a saber:

- Tendencia al orden en los sistemas abiertos relacionados con la teoría de las estructuras disipativas ya referidas anteriormente y trae la idea de la necesidad del cambio, del desequilibrio, del conflicto y de la reorganización, a través de un continuo movimiento generador de fluctuaciones y nuevas conexiones, por lo que las partes se reorganizan y forman nuevas entidades de orden superior.

- Ontología Sistémica viene a ser cuando las partes de una realidad conforman una totalidad organizada en estrecha interacción, cuya interpretación requiere el conocimiento tácito del sujeto que implica un enfoque interdisciplinario y los principios holográficos, según los cuales se supera la visión fragmentaria de las realidades y sus relaciones de causa y efecto para ir hacia ideas no lineales y eventos multicausales.

2 Según Franco y Dieterich (1999), el mundo del hoy se caracteriza por la complejidad de los problemas que a diario debe enfrentar y resolver, los cuales pueden ir de lo simple a lo complejo en peldaños sucesivos de complejidad que van desde el mecánico, al físico, el químico y el biológico hasta llegar al social, peldaño de más alta complejidad, propio de las organizaciones humanas constituidas por sujetos capaces de modificarlas y ser modificados por ellas. 
El trabajo como fenómeno de autoorganización

Mendoza de Ferrer, Hermelinda y Rodríguez P., María

- Conocimiento personal, según este postulado el sujeto construye la realidad por cuanto le da forma, moldea o estructura conforme a sus modos de hacer personales, categorías o características idiosincrásicas, por lo cual los datos activan bloques de conocimientos, moldeando lo que entra por los sentidos y la interpretación que se obtiene. Por ello el conocimiento es el resultado de un diálogo entre el sujeto y el objeto observado.

- Metacomunicación del lenguaje total coloca frente a la riqueza del espíritu humano y su capacidad para reflexionar acerca de sí mismo, criticarlo más allá de la simple expresión de la palabra y analizar su propio proceso, producir conocimiento y comunicarlo a sus semejantes. La comunicación que acompaña el mensaje, generalmente no verbal, cambia la esencia, expresa con exactitud, complementa y, especialmente da sentido al mensaje.

- Principio de complementariedad, que considera la necesidad de integrar en un todo coherente y bien pensado, los aportes provenientes de distintas personas, filosofías y métodos, a fin de captar la realidad en su justa dimensión y con toda la riqueza que ello implica para visualizarla desde una perspectiva sistémica. En lo cual, el diálogo e intercambio con otros espectadores, especialmente los ubicados en el bando contrario, constituye un aspecto clave para enriquecer la propia percepción.

\section{El sentido dialógico del trabajo}

En la situación de transitoriedad dada por el advenimiento de un paradig- ma que emerge yuxtapuesto a un sistema de ideas reduccionista, como el que se erige en el paradigma de la simplicidad, es preciso destacar el hecho de la complejidad implícita en el mundo del trabajo, al poder ser observado como objeto $\mathrm{y}$, simultáneamente, como sujeto que cuenta con la posibilidad de modificarse a sí mismo al darse el diálogo entre sujeto y objeto observado. Estas ideas son argumentadas por Martínez (1997) cuando hace referencia a la construcción de la realidad en función del conocimiento personal o Navarro (1997) cuando habla de las sociedades humanas como objetos que, sin perder su esencia, son al mismo tiempo colección de sujetos capaces de conocimiento y orientados a la acción transformadora, cuyos resultados son aparentemente distintos a la causa que los genera, aunque la reproducen completamente.

Partiendo de esta dinámica de la relación sujeto/objeto y profundizando el análisis de este aspecto, el trabajo ha experimentado como tendencia en el contexto emergente, una reconciliación de su carácter dual (sujeto-objeto; mente-mano; trabajo-ocio), en otras palabras, el trabajo como proceso esta orientado a la objetivación (creación de objetos), pero a su vez, en ese proceso de transformación de la naturaleza a través del trabajo, el hombre se transforma así mismo y se enriquece en términos de personalidad, valores, ideas, conocimiento y cultura, siendo esté el dialogo que se da en las dos dimensiones del fenómeno, la material e inmaterial.

En este sentido, las organizaciones y el trabajo no sólo son objetos complejos, sino también confluyen en su dinámica, una colección de sujetos cognitivos y pragmáticos capaces de modificar esas 
realidades (objeto) y modificarse así mismos como sujeto, de manera simultánea. A este proceso recursivo se le suma otro nivel de complejidad, por cuanto que, como seres humanos se tiene la posibilidad de intervenir objetos que también son sujetos, es decir, impactar la organización desde el exterior por ser ésta un sistema abierto no lineal donde el desequilibrio puede constituirse en energía transformadora, de allí que el desarrollo organizacional no sea un acto puntual, sino la transformación global la comunidad que constituye la organización.

Desde esta perspectiva, el trabajo, como hecho social e histórico que vincula las complejas relaciones sociales que ocurren en las organizaciones, adquiere dimensiones operacionales devenidas de fuentes diversas que confluyen en el contenido asumido y develan una serie de implicaciones en función de sus formas de organización y de la interpretación asignada por los sujetos que toman parte en el mismo que, como sujetos pueden transformarlo $y$, como objetos, ser transformados por él, es decir, el trabajo es producido por sujetos, quienes a su vez resultan producidos por éste, posición, según el Instituto de Estudios Avanzados para las Américas (Instituto de Estudios Avanzados para las Américas, INEAM, 2004), es diametralmente opuesta a la asumida por la Organización Científica del Trabajo cuya premisa era separar el trabajo de la persona del trabajador, esfuerzo que tomó la forma de mecanización del trabajador.

Al confrontar las interpretaciones que ocurren dentro de una realidad particular y entre diversas realidades, pueden evidenciarse disonancias que se constitu- yen en el ojo del huracán al convertirse en fuente de conflicto entre los postulados del paradigma tradicional y los del paradigma emergente, como también, entre la necesidad de dar respuesta y la capacidad para responder a las nuevas exigencias, en un mundo signado por la complejidad. Tal es el caso del mundo del trabajo y el empleo como forma de obtener los bienes y servicios necesarios para satisfacer las necesidades de la población, cuya sinergia debería articular los aspectos económicos e instrumentales de satisfacción de necesidades materiales con los propiamente culturales y relacionales de satisfacción de necesidades espirituales e intersubjetivas (INEAM, 2004).

En este orden de ideas es necesario preguntarse lo siguiente: ¿Cómo los sujetos del trabajo están transformando el trabajo?, ¿Cómo las nuevas formas de organización del trabajo están produciendo nuevos sujetos del trabajo?, ¿Cómo esa red de objetividades y subjetividades, de sujetos y objetos que se producen unos a otros no degenera en caos en las organizaciones? ¿Cómo logran formas elaboradas de estabilidad y capacidades de autoorganización a través de los modelos de producción?

Para dar respuesta a estas interrogantes, se presentarán algunas evidencias teóricas y empíricas de los postulados del paradigma emergente en el mundo del trabajo, para luego reflexionar acerca de sí el desequilibrio existente puede dar lugar o no a nuevas realidades con capacidad para responder a la necesidad de adaptación enfrentada por las organizaciones, ante el incremento de la complejidad del mundo que las rodea y la poca efectividad que están demostrando 
El trabajo como fenómeno de autoorganización

Mendoza de Ferrer, Hermelinda y Rodríguez P., María

tener las formas tradicionales de organización del trabajo, como consecuencia de la situación de transitoriedad que están confrontando al enfrentarse al agotamiento del sistema de pensamiento que les había dado sustento, según lo cual, la experiencia ya no es una guía útil para tomar decisiones por lo que deben reaprender a interactuar en un mundo signado por la complejidad, ambigüedad e incertidumbre.

\section{Postulados del paradigma emergente y su isomorfismo teórico-práctico en el mundo del trabajo}

El trabajo como fenómeno de autoorganización muestra el camino hacia una nueva manera de trabajar en equipo, más abierta y adaptable, en la que los individuos se manejen en límites claros al desenvolverse bajo modelos de comportamiento organizacional de tipo colegiado $^{3}$ (Davis y Newstrow, 1993). De allí que la idea del control, la comunicación, la información, y condicionantes del entorno, se estructuran como atractores que ayudan a crear y mantener en forma estable patrones y configuraciones dentro del sistema que, según Prigogine (1996) se convierte en generadores de inestabilidad que incrementa la entropía pero sólo hasta el límite de la manipulación del mundo del trabajo que genera una fuerza de choque capaz de originar nuevas reali- dades que permitan superar la entropía y superar el desequilibrio.

De allí, que los modelos de gestión, las formas de organización de trabajo (taylorista, fordista, toyotista, producción flexible, entre otros), la administración y los paradigmas, se conforman en mecanismos de autoorganización de las realidades, que se orientan a dar respuesta a los procesos de desequilibrio producto de los cambios e impacto del contexto, estableciendo los parámetros y límites que tienden a dar respuesta a los cambios y disipación del sistema social y en particular al sistema laboral.

En atención a estos planteamientos, los contenidos que se encierran en los paradigmas que se desarrollan alrededor del trabajo, permiten evidenciar los mecanismos a autoorganización que están contenido en los mismos.

En este sentido partiendo de la dinámica desarrollada alrededor del trabajo durante el siglo $X X$, se observa que se puso el énfasis en el apego a la ingeniería, por lo cual la tendencia fue a optimizar procesos para aumentar la productividad con un enfoque basado en la Gerencia Racional Científica. Sin embargo, esta perspectiva eminentemente "productiva", dejaba de lado el enfoque social y el de análisis de la problemática que se da con ocasión de la aplicación de los parámetros de ingeniería a los procesos de trabajo. Partiendo de esta realidad el elemento autoorganizador desde esta perspectiva

3 El modelo de comportamiento organizacional de tipo colegiado depende de la sociedad, la orientación de la gerencia es hacia el trabajo en equipo, la orientación del empleado es la responsabilidad y su resultado psicológico la autodisciplina (Davis y Newstrom, 1993). 
es la racionalidad mecanicista, que busca organizar el trabajo bajo la fórmula de la división y racionalización de los tiempos y movimientos para hacerlo más productivo, sin embargo, su agotamiento para lograr mejoras en la productividad genera un desequilibrio que hace surgir nuevas formas de comportamiento organizacional. De esta manera emerge la gerencia Cualitativa Humanística (Hickman y Silva, 1992) y la sociología del trabajo con sus distintos paradigmas como "respuesta a la demanda social generada por los ingenieros" (Guerra, 2001:73).

Continuando con el referido autor, en este contexto, aparece un conjunto de paradigmas que marcan el norte en el ámbito del trabajo, a saber: el adaptativo, de la modernización, francés humanista, clasista en el proceso de trabajo, la escuela de los radicals y los mercados segmentados y el de la especialización flexible.

El Paradigma Adaptativo, que busca mejoras en la productividad empresarial a la par de las condiciones de trabajo de sus integrantes. Para después de la Segunda Guerra Mundial el punto de interés se centraba en la necesidad de desarrollar la industrialización en países del tercer mundo y es cuando aparece el llamado paradigma de la modernización que se sitúa en las nociones macrosociales y macroeconómicas de las sociedades a fin de encaminarlas hacia el desarrollo. Uno de los paradigmas con más impacto en el enfoque social del trabajo es el francés humanista, donde resaltan los trabajos de George Friedmann citado por Guerra (2001), quien proponía "rescatar al trabajador obrero como actor fundamental en el proceso de trabajo" (Gue- rra, 2001:76), en el ámbito del modelo de desarrollo capitalista.

Tras la crisis producida por el agotamiento del modelo fordista de producción en masa surge el llamado paradigma clasista en el proceso de trabajo, orientación desarrollada por Harry Braverman citados por Guerra (2001), quien planteaba la disminución de la fuerza de las calificaciones obreras en beneficio del capitalismo monopolista y, por ende, una pérdida de las calificaciones profesionales, enfoque adoptado por Benjamín Coriat (1992), quien desarrolla un abordaje de los paradigmas productivos taylorismo, automatización y toyotismo. Retomando a (Guerra, 2001), la escuela de los "Radicals" y los mercados segmentados introduce la discusión sobre dualización, segmentación y polarización del trabajo. Todos estos aspectos refuerzan la dinámica de organización del trabajo en función de los paradigmas productivos, del capitalismo monopolista y de la división y segmentación de los mercados, todo ello producto de esfuerzos teórico-prácticos de dar control, límites y estructura a las múltiples manifestaciones asociadas con el trabajo productivo.

De forma más reciente, aparece el paradigma de la especialización flexible, planteado por Piore y Sabel citados por Guerra (2001), quienes, partiendo de la revisión del debilitamiento del modelo fordista, con evidencias empíricas, muestran cómo las organizaciones menos afectadas por la crisis económica de los 80’ fueron aquellas que tenían "formas de producción más flexibles; que sabían adaptarse con más facilidad a los constantes vaivenes y fluctuaciones de la economía" (Guerra, 
El trabajo como fenómeno de autoorganización

Mendoza de Ferrer, Hermelinda y Rodríguez P., María

2001: 78), dando lugar al análisis y propuestas de formas de trabajo más flexibles, como también al análisis de diferentes formas de regulación y de los sistemas de relaciones laborales que les dan sustento.

El agotamiento del paradigma taylorfordista trajo consigo la necesidad de abordar nuevos modelos orientados a lo que se supone es una nueva forma de obtener mejoras en la productividad caracterizada de la siguiente manera:

1. Polivalencia en materia de calificaciones.

2. Flexibilidad en cuanto al uso del tiempo de trabajo y movilidad de los trabajadores.

3. Flexibilidad productiva de los medios de trabajo para adaptarse rápidamente con el objeto de producir series cortas de productos heterogéneos.

4. Esfuerzo de investigación y desarrollo para innovar en cuanto a los procesos y productos.

5. Técnicas japonesas de organizar las empresas trabajando "justo a tiempo" y en redes con subcontratistas y proveedores (Neffa, 1999).

En línea con lo hasta ahora planteado, Novick (2000: 116) encuentra que, lo socialmente impactante de "el trabajo", son las formas de organización que se generan alrededor del mismo, las cuales suponen, un "conjunto de aspectos técnicos y sociales que intervienen en la producción de determinado objeto". Se refiere a la división del trabajo entre las personas y las máquinas donde intervienen el medio ambiente y la totalidad de las dimensiones presentes en cualquier prestación laboral.
Estas formas de organización del trabajo, se dan alrededor de los llamados modelos de producción o sistemas productivos. En este sentido, para poder analizar los aspectos que definen al trabajo y las formas de organización alrededor del mismo, es necesario precisar los rasgos que caracterizan a los sistemas productivos y los paradigmas productivos.

\section{Sistemas y paradigmas productivos}

Para hacer el abordaje de dichos aspectos Boyer y Duran citados por Lucena (2003:71), plantean un acercamiento a la definición, estableciendo que se entiende por Sistemas Productivos: "La complementariedad y coherencia entre los principios de gestión, la organización interna de la firma, su articulación con las empresas subcontratistas y con la competencia y la relación salarial... y el sistema educativo sin olvidar la regulación macroeconómica, constituyen los elementos que configuran el sistema productivo".

En este sentido, Neffa citado por Lucena (2003) propone una clasificación de los sistemas productivos donde muestra la evolución histórica acaecida en ocasión de la necesidad de obtener beneficios a través de mejoras en la productividad, capacidad para atender satisfactoriamente el mercado, estimulación del consumo e incorporación del saber científico y tecnológico, para lo cual parte desde los sistemas productivos más elementales y rudimentarios, hasta los más sofisticados, incluyendo una categoría propia de la era digital. Cada una de los cuales va a impactar la organización del trabajo y 
las relaciones que surgen dentro del mismo, siendo estos el artesanado, la manufactura y el maquinismo.

Vemos entonces como, en los sistemas del trabajo todo está en un continuo movimiento generador de desestabilización y de nuevas relaciones, en las cuales los componentes del trabajo se reorganizan para formar nuevas realidades y modos de organización, donde se enfatizan diferentes aspectos según su orientación; convirtiendo la inestabilidad en punto focal de la transformación y el desequilibrio en fenómeno de autoorganización para mantener el orden en los sistemas abiertos que interactúan en el mundo del trabajo, como proceso abierto en espiral.

Ahora bien, Neffa citado por Lucena (2003) ve el trabajo humano como una realidad compleja que adquiere diversas dimensiones como serían la del trabajo como tarea para conocer y dominar el universo, a fin de arrancarle sus riquezas. Como ocasión privilegiada para tomar conciencia de las debilidades y potencialidades humanas y su potencial para el desarrollo de la personalidad, si el mismo se realiza en condiciones y medio ambiente adecuados. Como hecho social al ser parte de una realidad colectiva para generar los bienes y servicios que la especie humana requiere para perpetuarse, dimensión que lleva implícito un comportamiento solidario a partir de la constitución de un colectivo de trabajo y, finalmente su dimensión de trascendencia, al incitar al proceso de construcción de una sociedad, así como a satisfacer las necesidades de sus miembros para la libertad y el ejercicio de sus derechos personales y sociales, planteamiento compartido por Sen citado por Schtulmann (S/F).

Candelero (2001), cuestiona la concepción económica del trabajo que sustituye los vínculos desarrollados entre los miembros de una comunidad moral, por aquellos que lo hacen dependiente de la actividad productiva. Según esa concepción, el trabajo es para el mercado y se ve reducido a lo que comúnmente se ha dado en denominar empleo. De esta forma, el trabajo se deshumaniza, el trabajador se convierte en su objeto y la persona es valorada sólo en la medida en que le es útil, de allí la gran angustia que genera, en la sociedad industrial, no encontrar empleo o sencillamente perderlo.

Ante esta situación, Lucena (1999: 148 -149) considera que "los procesos de reestructuración empresarial que se expresan en un continuado achatamiento, externalización, adelgazamiento de las organizaciones, evidentemente vienen reduciendo las nóminas de las empresas, y debilitando las organizaciones sindicales. La expulsión de trabajadores de empresas grandes para empresas pequeñas, igualmente se convierte en mecanismo debilitante por la poca acción sindical en estas últimas. Hoy asistimos a la existencia de un déficit dramático y creciente en el surgimiento de los empleo. Sin embargo, los ciudadanos y la población en general, de alguna manera tienen que sobrevivir. Es por lo que el paradigma del empleo asalariado que permite a una persona disponer de un salario y de una protección legal laboral, está cada vez más distante de las nuevas generaciones. Se señala que entre las respuestas a esta situación, está el desarrollo de la empresarialidad." 
El trabajo como fenómeno de autoorganización

Mendoza de Ferrer, Hermelinda y Rodríguez P., María

En la mayoría de los países latinoamericanos el desarrollo de la empresarialidad va unido al crecimiento de la informalidad y la exclusión, en tal sentido, un punto en el que invita a reflexionar Alonso (1992: 126), ubicándose en el contexto del porfordismo, es el relativo a la vuelta e indetenible ascenso del "homo economicus" a partir de la crisis económica de los años setenta cuando el bien común comienza a verse seriamente afectado por cuanto que la afectividad por sí mismo y la máxima rentabilidad pasan a ser el motor impulsor de las acciones y la inseguridad del mercado de trabajo la forma de controlar el costo y disponibilidad del ejército de reserva en el que se convirtió a la fuerza de trabajo, al abandonar las políticas de pleno empleo "y con ello la contención de las demandas salariales, el desempleo masivo, la intensificación del uso del factor trabajo contratado y el desarrollo de políticas de oferta". Razón por la cual plantea la necesidad de un nuevo pacto social o tercer paradigma, capaz de reconciliar los paradigmas precedentes.

En contraste con estas dimensiones Juan Pablo II (1981), afirma que, además de proveer de sustento a quien lo realiza, contribuye al desarrollo de la ciencia y la técnica, así como a elevar la cultura y moral de la sociedad donde se realiza e indica que "'trabajo" significa todo tipo de acción realizada por el hombre independientemente de sus características o circunstancias; significa toda actividad humana que se puede 0 se debe reconocer como trabajo entre las múltiples actividades de las que el hombre es capaz y a las que está predispuesto por la naturaleza misma en virtud de su humanidad".
Los autores citados dan cuenta de cómo el trabajo no puede ser estudiado de una manera aislada, por cuanto que, en el nuevo paradigma productivo, sus partes conforman una totalidad organizada en estrecha interacción entre sí, cuya complejidad amerita un enfoque interdisciplinario y la superación de la visión fragmentaria de las realidades que lo integran y de las relaciones de causa y efecto para buscar su interpretación desde ideas no lineales y eventos multicausales, propios de la ontología sistémica y que, una vez más se evidencia como ante la presencia de desequilibrios aparece la autoorganización, en este caso expresada por la empresarialidad expuesta por Lucena (2003) y el nuevo pacto social propuesto por Alonso (1992).

Viendo desde otro ángulo este complejo mundo, puede observarse que el trabajo tiene un sentido objetivo en cuanto que es una acción realizada por el hombre y otro subjetivo por cuanto que, el hombre posee inteligencia y voluntad que lo capacitan para actuar en forma racional y programada, como también, para decidir acerca de sí y tender a su realización. Siendo de esta manera, el hombre sujeto del trabajo. Como persona trabaja, realiza acciones correspondientes al proceso de trabajo, las cuales, independientemente de su contenido objetivo, van a contribuir a la realización de su humanidad, la de su familia y de la sociedad a la cual pertenece, dándole así una nueva dimensión ética al trabajo al valorizar su dimensión subjetiva y con ello al hombre como sujeto del mismo (Juan Pablo II, 1981).

En cambio, para Carrillo e Iranzo (2000), un punto a considerar en la emergencia de un nuevo paradigma producti- 
vo tiene que ver con la discusión teórica acerca de la calificación en el trabajo. Aspecto que ha transcurrido desde los clásicos quienes denuncian un deterioro progresivo de la calificación individual integral para ceder el paso a un saber obrero segmentado o a una expropiación del saber obrero, pasando por la sociología del trabajo francesa con sus posiciones antagónicas en torno al cambio técnico y su impacto en la organización y la calificación del trabajo, hasta el intento por rescatar los conocimientos tácitos, no reconocidos, para el funcionamiento de los sistemas productivos, que ponen de relieve el rol desempeñado por el trabajo obrero para prever, identificar y solucionar problemas, actividades específicas de la calificación, que suponen capacidad cognoscitiva.

Como resultado de lo expresado en los párrafos precedentes, desde mediados de los ochenta, ante la necesidad de confrontar la incertidumbre involucrada en el proceso productivo al verse la organización sometida a la exigencia de movilización de conocimientos y saberes diferentes, se ha comenzado a emplear el término competencias, cuya singularidad respecto al de calificación, utilizado durante el paradigma tradicional, radica en que designa saberes de diferente orden, anteriormente no considerados, remite a trayectorias y atributos individuales y da cuenta de una nueva reestructuración de los trabajadores diferenciándolos según sus funciones y niveles de competencia y unificándolos por objetivos y comportamientos comunes.

De esta forma, Juan Pablo II (1981) al incorporar la dimensión ética del trabajo y Carrillo e Iranzo (2000) al considerar la calificación en el trabajo, como sujetos que estudian el trabajo, construyen la realidad dándole forma, moldeándola o estructurándola conforme a sus modos de hacer personales, categorías o características idiosincrásicas, en diálogo con el trabajo como objeto observado, para producir el conocimiento personal cuando, lo que les entra por los sentidos es contrastado con sus referentes y al hacerlo producen nuevas realidades convirtiéndose en sujetos y objetos, ya que, en palabras de Navarro (1997:2) "el sujeto no puede contemplar su acción en el proceso de recaer sobre sí mismo por vía del objeto, y el conocimiento de ese objeto no puede entenderse como algo que efectivamente trastoca las condiciones de ese conocimiento en el sujeto." Situación que ubica a los estudios del trabajo en un marco de complejidad del objeto y de los sujetos, sus mentes y conciencias, cuya interacción constituye el medio social específico de nuestra especie y complejidad que, según Prygogine (1996:185), tiene sus límites en el campo del trabajo por cuanto que aunque "puedo fácilmente transformar el trabajo -energía llamada organizada- en calor, es difícil e incluso a veces imposible transformar el calor en trabajo", sin embargo, quienes lo realizan o coordinan están en constante movimiento para alcanzar los objetivos propuestos y ese movimiento tiene la posibilidad de producir nuevas realidades.

Tomando otra arista en el ámbito del trabajo, De la Garza (1999) puntualiza las nuevas tendencias en los modelos de producción y algunas características claves de los mismos, los cuales se asumen como nuevas expresiones de autoorganización. Dando cuenta de que no se trata 
El trabajo como fenómeno de autoorganización

Mendoza de Ferrer, Hermelinda y Rodríguez P., María

de un modelo único, sino por el contrario de varias formas de abordar el problema de la productividad entre los cuales incluye, además de los postfordistas, el toyotismo, lean producction, los nuevos conceptos de producción, el sistema de manufactura, el industrial governace y las nuevas relaciones industriales.

En cambio, Coriat (1992: 179) indica que las innovaciones organizacionales producen cambios en el uso y formas de "consumo productivo del trabajo vivo", como es el caso del modelo general emergente cuyos contornos describe en relación al conjunto de efectos que redefinen la configuración de los grupos de trabajo, siendo estos:

1. Un efecto de desplazamiento si se limita sólo al trabajo concreto.

2. Un efecto de reclasificación, referido a la valoración o descalificación de habilidades de diferentes categorías socioprofesionales.

3. Un efecto de reorganización que hace emerger figuras renovadas 0 inéditas del sistema productivo postayloriano aún en gestación.

De estas modificaciones parece que surge un nuevo modelo de trabajo con potencialidad de ponerse en funcionamiento en modalidades opuestas. Un modelo de trabajo formado a partir de la concepción de obreros marginados, detectores y minimizados sometidos a estrictas relaciones de jerarquía y el otro donde la productividad de la empresa se obtiene mediante la construcción de tríadas entre fabricantes, tecnólogos y administradores, cada una de las cuales presenta costos y beneficios, aunque sean injustamente pagados por los actores involucrados.
En este caso el fenómeno de autoorganización se evidencia en De la Garza (1999) al referirse a las tendencias en los modelos de producción y en Coriat (1992), al reseñar los efectos de las innovaciones organizacionales respecto al desplazamiento, la reclasificación y reorganización que consideran la redefinición de los grupos de trabajo frente a la riqueza del potencial humano y su capacidad para reflexionar acerca de sí mismo y el trabajo que realiza, cuestionarlo y analizar su propio proceso para encontrar nuevas e innovadoras formas de realizarlo. En consecuencia, la metacomunicación del lenguaje es fuente de conocimiento que puede ser comunicado a los semejantes para alcanzar un conocimiento cada vez más sofisticado que contribuya al mejoramiento continuo de la organización, hasta niveles de utopía, denominado por Gergen y Tojo (1996) crecimiento estable. Esto quiere decir que la tendencia actual del trabajo asociado a la incorporación e intervención de las tecnologías de la comunicación e información en los procesos, transfiere al lenguaje una relevancia trascendente como mecanismo de intercambio a través de símbolos, ideas, imágenes entre otros, que lo configura como elemento de autoorganización y autoorganizador.

\section{Una mirada a la realidad de América Latina y Venezuela}

A fin de encontrar intersticios por donde se cuela el principio de complementariedad en un espacio territorial específico, demos una breve mirada a Latinoamérica, región que presenta una realidad particular en relación al mejoramiento continuo de la organización y sus 
implicaciones en el mundo del trabajo, pues, desde hace más de una década busca escapar de una crisis en espiral que socava la trama social, hunde en el marasmo a sus economías y debilita las bases de sus democracias al profundizar la deslegitimación de sus instituciones, donde también está presente el factor trabajo, acusándolas de incapacidad para atender los problemas del desempleo, discriminación de los mercados de trabajo, destrucción del poder adquisitivo de los trabajadores e incremento de la pobreza, para lo cual Villanueva (2003) propone el desarrollo humano como forma de contrarrestar la agobiante crisis.

Por su parte, Lucena (2003), al analizar la situación de las relaciones de trabajo en Latinoamérica, afirma que las transformaciones experimentadas por los modelos políticos para dirigir las sociedades favorecieron opciones conservadoras del modo de hacer u orientaciones de carácter neoliberal, apoyándose en el argumento de que el modelo de desarrollo basado en la sustitución de importaciones, en conjunto con las tendencias populistas, fue el causante de la hipertrofia del Estado volviéndolo inútil para cumplir con sus obligaciones. Es así como, en los sesenta, se produce la primera crisis económica del sistema democrático venezolano, luego del crecimiento explosivo con sobredimensionamiento económico del 74 al 82, para arribar a 1983 cuando el cierre del mercado cambiario y el establecimiento del Régimen de Cambios Diferenciales mostraron la punta del iceberg de la crisis e inicio de la recesión que se mantiene veinte años después (FUNDAFUTURO, 1992).
En palabras de Lucena (2003:148), a lo largo de los modelos económicos y políticos vividos en la Región se construyó una red de relaciones caracterizada por la heterogeneidad, puesto que, los contrastes existentes entre sectores, regiones e instituciones, determinaron desigualdades, profundizadas con la aplicación de las políticas neoliberales, expresadas a inicios de los noventa mediante la apertura y las políticas integracionistas que, si bien es cierto que apuntalaron algunos sectores, también arrastraron a otros a la crisis, en función de las simetrías y asimetrías existentes entre ellos ante los desafíos de la integración, en un mundo globalizado donde el capital internacional tomó ventaja. Afirma que "Siempre han existido factores externos participando de alguna manera en las relaciones económicas de las sociedades, mucho más en nuestra condición de sociedades dependientes. Pero la antigua división del trabajo en el ámbito mundial que nos colocaba en el papel de economías cerradas, que ofertaban al mundo desarrollado sólo materias primas, daba pie a dos sistemas diametralmente diferentes en las relaciones internacionales. Con la globalización, nos llegan productos de todo tipo y podemos enviar los nuestros a cualquier parte, claro que los niveles de desarrollo entre los países y sus respectivas economías, determinan que seguimos enviando materias primas y recibiendo productos de mayor valor agregado. Con la globalización no ha cambiado tanto la división internacional del trabajo, pero si han cambiado los conceptos y condiciones de funcionamiento de las relaciones económicas internacionales." 
El trabajo como fenómeno de autoorganización

Mendoza de Ferrer, Hermelinda y Rodríguez P., María

Continuando con este autor, en el caso venezolano, no es sino a partir de 1958 cuando ocurre el desarrollo industrial, caracterizado por un acelerado proceso de formación del movimiento obrero, con importante intervención político partidista, la magnitud y desarrollo de las compañías petroleras y un Estado dependiente de la renta petrolera. La estructura económica se diversificó al implementarse el modelo de Sustitución de Importaciones que ampliaba el proceso de industrialización, el Estado amplió los servicios públicos, sin embargo en el contexto laboral, en 1959, 1960, 1961, 1962 y 1969 ocurren en el país 15, 36, 14, 19 y 86 huelgas, respectivamente, que dan cuenta de los desequilibrios existentes.

En la década del setenta las huelgas tendieron a desaparecer, lo que no supuso la ansiada paz laboral que el sistema exigía; sirviendo las convenciones colectivas como medio para mejorar las condiciones de trabajo y de vida, así como, el acuerdo entre empresarios y trabajadores para mantener la paz laboral (Lucena, 1992). De esta manera el equilibrio del sistema se lograba mediante proceso de negociación de tipo distributivo, en una relación gana-pierde donde jugaban un papel importante las relaciones de poder y el mantenimiento del status quo, en detrimento del bien común. Situación que persiste hoy en día y que, a la larga afecta a las organizaciones y su capacidad de autoproducirse en un proceso dialógico, según el cual capital y trabajo, empresa y trabajador, siendo antagónicos, colaboran entre sí y "producen la organización y la complejidad" (Morin, 1990: 106). Tal como el remolino es simultáneamente producido y productor.
A partir de los planteamientos formulados por Coriat (1992) Villanueva (2003) y Lucena (1992) se hace presente la necesidad de adoptar el principio de complementariedad, a fin de captar en su justa dimensión y con toda la riqueza que ello implica al mundo del trabajo y visualizarlo desde una perspectiva sistémica. Por ello, resulta imprescindible el diálogo e intercambio entre varios estudiosos o investigadores, especialmente los ubicados en posiciones contrarias de forma que se pueda enriquecer la propia percepción pues, tal como lo establece Lucena (2003:29), "las dimensiones del trabajo son muy variadas, lo que demanda que su estudio implique disciplinas muy diversas."

Por su parte, Sen (2001) considera "que la principal fuente de esperanza en la posible armonía en el mundo contemporáneo radica en la pluralidad de nuestras identidades, que se superponen unas a otras y se oponen a divisiones marcadas por una línea única y endurecida de impenetrable división" como lo son los sistemas de clasificación por categorías que aspiran arroparlo todo reduciendo a la gente a una sola dimensión definida por el sistema dominante, a diferencia de la diversidad plural como factor de unificación al favorecer una interacción más estrecha que posibilita el análisis de la realidad desde perspectivas complementarias y enriquecedoras.

\section{Conclusiones}

El trabajador, como ser social, transforma la realidad y al mismo tiempo es transformado por ella en un proceso de interretroacción y recursividad que incluye los problemas complejos que tras- 
cienden la relación causa-efecto, como es el caso de los problemas humanos, cuya complejidad puede ser explicada a partir de los principios holográficos al considerar la presencia del todo en las partes y de las partes en el todo, el hombre está en la realidad y ésta está en el hombre pues todos formamos parte de una misma red.

En la bibliografía consultada se encuentra que las realidades sociales, propias del mundo del trabajo, se estructuran mediante estilos de organización afines al holográfico, ya que, desde este punto de vista, los sujetos individuales no mimetizan la organización sino que la constituyen, no son miniaturas de la organización sino causa de la misma; de allí que no exista un todo organizacional, sino tantas versiones como sujetos individuales lo postulan, para ser constantemente reconstruido según los procesos de disipación de la intencionalidad de los actores en constante interacción. Vista de esta manera, la disipación es un fenómeno creativo, que en lugar de incrementar el desorden, hace surgir nuevas estructuras no sometidas directamente al control de los sujetos que inducen su aparición. Es así cómo los sujetos del trabajo lo están transformando a partir de los desequilibrios emergentes que se constituyen en energía creativa para dar lugar a nuevas realidades.

De esta manera se logran formas elaboradas de estabilidad y se desarroIlan capacidades de autoorganización y, a su vez, las nuevas formas de organización están produciendo nuevos sujetos del trabajo al producirse un proceso de interretroacción cuando el sujeto se convierte en objeto al transformar el trabajo $\mathrm{y}$, simultáneamente ser transformado por éste en un sistema de objetividades dadas por el trabajo que se realiza y de subjetividades expresadas en la interpretación que del mismo se hace para constituirse en una red de sujetos y objetos que se producen unos a otros.

Ahora bien, esta red no degenera en caos, como se mencionó anteriormente; las organizaciones, a partir de sujetos que las conforman son capaces de aprendizaje y se orientan a una acción transformadora, que sin hacerles perder su esencia, impulsa la producción de nuevas realidades cuyos resultados aparentemente distintos las reproducen completamente ratificando que las organizaciones, además de objetos complejos, son también colección de sujetos capaces de modificarlas $y$, simultáneamente, ser transformados por ellas mediante el diálogo e intercambio en la relación sujeto-objeto capaz de producir la autoorganización a partir del caos y la confusión, tal como podría ocurrir en las nuevas formas de organización del trabajo donde, necesariamente surgirán nuevas formas de conformación de los actores productivos.

De allí que los postulados que emergen en los paradigmas, los modelos de producción aportados como mecanismos de organización y autoorganización del trabajo, los modelos de gestión y la administración, no son más que respuestas que buscan atender las realidades complejas del mundo de hoy. 
El trabajo como fenómeno de autoorganización

Mendoza de Ferrer, Hermelinda y Rodríguez P., María

\section{Referencias Bibliográficas}

Almarza, Fernando (2002), La Teoría del Caos. Hacia un Modelo Unitario de Interpretación Epistémica. Escritos en arte, estética y cultura. III etapa, número 15, Caracas, enero - junio 2002, pp.: 107-150.

Alonso, Luis (1992), Postfordismo, Fragmentación Social y Crisis de los Nuevos Movimientos Sociales. Sociología del Trabajo. Revista cuatrimestral de empleo, trabajo y sociedad. Sistemas de producción complejos en España y Portugal. Otoño de 1992. Nueva época: Madrid.

Candelero, Manuel (2001), El Impacto Tecnológico: Trabajo Decente o Vida Decente. Ponencia oficial para el VIII Congreso Nacional del Equipo Nacional del Trabajo. Catamarca, Mayo de 2002.

Carrillo, Jorge e Iranzo, Consuelo (2000), Calificación y Competencias Laborales en América Latina. De la Garza, E. (Ed) Tratado Latinoamericano de Sociología del Trabajo. Editorial Fondo de Cultura Económica. México, pp. 179211.

Coriat, Benjamín (1992), El Taller y el Robot. Ensayos sobre el Fordismo y la Producción en Masa en la Era de la Electrónica. Siglo XXI Editores: México.

Davis Keith y John Newstrom (1993), Comportamiento Humano en el Trabajo. Comportamiento Organizacional. Mc Graw Hill: México.

Drucker, Peter (1993), La Sociedad Postcapitalista. Bogotá-Colombia: Norma.

De La Garza, Enrique (1999), Epistemología de las Teorías Sobre Modelos de Producción. Revista los retos teóricos de los estudios del trabajo hacia el siglo XXI. Colección Grupos de Trabajo de Clacsco. Buenos Aires, pp. 117- 156.

Franco Raimundo y Heinz Dieterich (1999), Hacia la Sociedad Post-Capitalista: la
Fase de Transición. En Heinz Dieterich y otros. Fin del Capitalismo Global. El Nuevo Proyecto Histórico. La Habana, Cuba: Ciencias Sociales, pp.81-114.

FUNDAFUTURO (1992), Cuando Venezuela Perdió el Rumbo. Un Análisis de la Economía de Venezuela entre 1945 y 1991. Caracas: CAVENDES.

Guerra, P. (2001), Sociología del Trabajo. 2da edición. Fundación Cultura Universitaria. Montevideo. Uruguay.

Gergen, Kenneth y Tojo, Joseph (1996), Organizational Science in Postmodern Context, Draft Copy for Journal of Applied Behavorial Science № 32, pp. 356-377.

Hickman, Craig y Silva, Michael (1992), Como Organizar Hoy Empresas con Futuro. Editorial Granica. Argentina.

Instituto de Estudios Avanzados para las Américas - INEAM (2004), Módulo 4 Ética y Capital Social: Contenidos Temáticos. En ¿Cómo Enseñar Ética, Capital Social y Desarrollo en la Universidad? BID-OEA [Consulta en línea]. Disponible en http://www.educoea. org. [Consulta: 28 de Octubre de 2004]

Juan Pablo II (1981), Laborem Exercens. Carta Encíclica sobre el trabajo humano en el 90 aniversario de la Rerum Novarum. [Consulta en línea]. Disponible en www.encuentra.com. [Consulta: 06 de OCTUBRE de 2004]

Lucena, Héctor (1992), El Movimiento Obrero Petrolero. Proceso de Formación y Desarrollo. El Centauro Ediciones. Caracas, Venezuela.

Lucena, Héctor (1999), El Enfoque de las Relaciones Industriales y los Estudios Laborales. En Garza, E. de la: Los Retos Teóricos de los Estudios del Trabajo Hacia el Siglo XXI. Buenos Aires, Argentina: Consejo Latinoamericano de Ciencias Sociales - CLAC- 
SO y Agencia Sueca de Desarrollo Internacional - ASDI, pp.: 141 - 156.

Lucena, Héctor (2003), Las Relaciones de Trabajo al Inicio del Nuevo Siglo. Fondo Editorial Tropykos. Caracas.

Martínez, Miguel (1997), El Paradigma Emergente. Hacia una nueva teoría de la Racionalidad Científica. México: Trillas.

Morin, Edgar (1990), Introducción al Pensamiento Complejo. Barcelona, España. Gedisa.

Navarro, Pablo (1997), La Metáfora del Holograma Social. Universidad de Oviedo. España. Disponible http://evunix.uevora.pt/ eje/hologramasocial. html. [Consulta: 2004, Septiembre 13]

Neffa, Julio César (1999), Crisis y Emergencia de los Nuevos Modelos de Producción. Revista los retos teóricos de los estudios del trabajo hacia el siglo XXI. Colección Grupos de Trabajo de Clacsco. Buenos Aires, pp.: 69-115.

Novick, Martha (2000), La Transformación de la Organización del Trabajo. De la Garza E. (Ed) Tratado Latinoamericano de Sociología del Trabajo. Editorial Fondo de Cultura Económica. México, pp. 123-147.

Prigogine,llya (1996), ¿Un Siglo de Esperanza? En Prigogine y otros. Ilya Prigogine: El Tiempo y el Devenir. BarceIona-España: GEDISA.
Schvarstein, Leonardo (1998), Diseño de Organizaciones. Tensiones y Paradojas. Buenos Aires, Argentina. Paidos.

Schtulmann, Alejandro (S/F), Comentario del libro Desarrollo y Libertad de Amartya Sen (2001) Documento incluido en la Biblioteca Digital de la Iniciativa Interamericana de Capital Social, Ética y Desarrollo, del Banco Interamericano de Desarrollo (BID). Disponible en http://www.iadb.org/etica. [Consulta: 2004, julio 31]

Sen, Amartya (2001), Exclusión e Inclusión. Documento incluido en la Biblioteca Digital de la Iniciativa Interamericana de Capital Social, Ética y Desarrollo, del Banco Interamericano de Desarrollo (BID). Disponible en http://www. iadb.org/etica. [Consulta: 2004, agosto 16].

Villanueva, Ricardo (2003), La Atención a las Necesidades de Aprendizaje como Abordaje Integrador del Desarrollo Humano. Documento incluido en la Biblioteca Digital de la Iniciativa Interamericana de Capital Social, Ética y Desarrollo, del Banco Interamericano de Desarrollo (BID). Disponible en http://www.iadb.org/etica. [Consulta: 2004, octubre 20] y en Desarrollo Humano e Institucional en América Latina (DHIAL), ISSN 1577-5232, № 45, 2003 http://dialnet.unirioja.es [Consulta: 2006, febrero 22]. 